\title{
MEROMORPHIC FUNCTIONS ON A COMPACT RIEMANN SURFACE AND ASSOCIATED COMPLETE MINIMAL SURFACES
}

\author{
KICHOON YANG
}

(Communicated by Jonathen M. Rosenberg)

\begin{abstract}
We prove that given any meromorphic function $f$ on a compact Riemann surface $M^{\prime}$ there exists another meromorphic function $g$ on $M^{\prime}$ such that $\{d f, g\}$ is the Weierstrass pair defining a complete conformal minimal immersion of finite total curvature into Euclidean 3-space defined on $M^{\prime}$ punctured at a finite set of points. As corollaries we obtain i) any compact Riemann surface can be immersed in Euclidean 3-space as in the above with at most $4 p+1$ punctures, where $p$ is the genus of the Riemann surface; ii) any hyperelliptic Riemann surface of genus $p$ can be so immersed with at most $3 p+4$ punctures.
\end{abstract}

\section{INTRODUCTION}

Let $M$ be a (connected) Riemann surface and consider a conformal minimal immersion $\varphi: M \rightarrow \mathbf{R}^{3}$. It is a fundamental theorem due to Chern and Osserman [CO] that for a complete $\varphi$ (i.e., the induced metric on $M$ is complete) the total curvature is finite if and only if the Gauss map is algebraic. (In fact this result is true in any $\mathbf{R}^{n}$. However, our interest lies solely in the case $n=3$.) For the sake of simplicity we shall call a complete conformal minimal immersion $\varphi: M \rightarrow \mathbf{R}^{3}$ of finite total curvature an algebraic minimal surface. In particular if $\varphi: M \rightarrow \mathbf{R}^{3}$ is an algebraic minimal surface then $M$ is, via a biholomorphism, identified with a compact Riemann surface $M^{\prime}$ punctured at finitely many points and the Gauss map of $\varphi$ extends holomorphically to all of $M^{\prime}$. Klotz and Sario [KS] proved that there exists an algebraic minimal surface of every genus. Hoffman and Meeks [HM] later exhibited an algebraic minimal surface of every genus with exactly three punctures that is actually embedded. On the other hand Gackstatter and Kunert [GK] proved that any compact Riemann surface can be immersed in $\mathbf{R}^{3}$ as an algebraic minimal surface with finitely many punctures.

In the present paper we prove that given any meromorphic function $f$ on a compact Riemann surface $M^{\prime}$ there exists another meromorphic function $g$ on $M^{\prime}$ so that $\{d f, g\}$ is the Weierstrass pair giving an algebraic minimal

Received by the editors July 11,1988 and, in revised form, August 1, 1988.

1980 Mathematics Subject Classification (1985 Revision). Primary 53A10; Secondary 53C42. 
surface defined on $M^{\prime}$ punctured at the supports of the polar divisors of $f$ and $g$. Since there always are an abundant supply of meromorphic functions on a Riemann surface our theorem implies the Gackstatter-Kunert theorem. As corollaries of our theorem we also obtain the following: i) any compact Riemann surface of genus $p$ can be immersed in $\mathbf{R}^{3}$ as an algebraic minimal surface with at most $4 p+1$ punctures, ii) any hyperelliptic Riemann surface of genus $p$ can be immersed in $\mathbf{R}^{3}$ as an algebraic minimal surface with at most $3 p+4$ punctures.

Our proof uses the Riemann-Roch theorem in an essential way and the technique is a variation on the ones used in [GK], [CG], and [BC].

\section{$\S 1$. The Weierstrass RePresentation Formula}

Consider a conformal minimal immersion $\varphi=\left(\varphi^{\alpha}\right): M \rightarrow \mathbf{R}^{3}$ from a Riemann surface $M$. The Gauss map of $\varphi$ is a map $M \rightarrow \mathbb{C} P^{2}$ given by

$$
\Phi: z \mapsto^{t}\left[\left(\frac{\partial \varphi^{\alpha}}{\partial z}\right)\right]
$$

where $z$ is a local holomorphic coordinate in $M$. The differential of $\varphi$ gives globally defined holomorphic 1-forms $\left(\zeta^{\alpha}\right)$ on $M$ given locally by

$$
\zeta^{\alpha}=\eta^{\alpha} d z, \quad \text { where } \eta^{\alpha}=\frac{\partial \varphi^{\alpha}}{\partial z} .
$$

We then must have

$$
\begin{gathered}
\Sigma\left|\eta^{\alpha}\right|^{2}>0 \\
\Sigma\left(\eta^{\alpha}\right)^{2}=0 \\
\text { the } \zeta^{\alpha} \text { 's have no real periods. }
\end{gathered}
$$

Condition (1) means that $\varphi$ is an immersion. Condition (2) provides that $\varphi$ is conformal. The holomorphy of $\left(\zeta^{\alpha}\right)$ then reflects the fact that $\varphi$ is minimal. Condition (3) says that the line integrals $\operatorname{Re} \int^{z}\left(\zeta^{\alpha}\right)$ are path independent. This is so since we must have

$$
\varphi^{\alpha}(z)=2 \operatorname{Re} \int^{z} \zeta^{\alpha}
$$

Conversely once we have holomorphic 1-forms $\left(\zeta^{\alpha}\right)$ on $M$ satisfying (1), (2), and, (3) then (4) defines a conformal minimal immersion $M \rightarrow \mathbf{R}^{3}$.

Assume now that $\varphi(M)$ does not lie in the $x y$-plane in $\mathbf{R}^{3}$. Introduce the holomorphic 1-form $\mu$ and the meromorphic function $g$ by

$$
\mu=\zeta^{1}-i \zeta^{2}, g=\zeta^{3} / \eta
$$

where $\mu=\eta d z$. Note that $\mu$ is a holomorphic 1 -form on $M$ and $g$ is a meromorphic function on $M$ such that whenever $g$ has a pole of order $m$ at a point then $\mu$ has a zero of order $2 m$ at the same point. (See [L], p. 113.) 
$\{\mu, g\}$ is called the Weierstrass pair of $\varphi$. Conversely given a pair $\{\mu, g\}$ on $M$ whose zeros and poles are related as mentioned above we may put

$$
\zeta^{1}=\frac{1}{2}\left(1-g^{2}\right) \mu, \quad \zeta^{2}=\frac{i}{2}\left(1+g^{2}\right) \mu, \quad \zeta^{3}=g \mu
$$

giving rise to holomorphic 1-forms $\left(\zeta^{\alpha}\right)$ on $M$ satisfying (1) and (2). It follows that $\left(\zeta^{\alpha}\right)$ defines a conformal minimal immersion at least on the universal cover of $M$. In order for $\left(\zeta^{\alpha}\right)$ to define a conformal minimal immersion on $M$ we must have the condition (3) met also.

Let $M=M^{\prime} \backslash \Sigma$, where $M^{\prime}$ is a compact Riemann surface and $\Sigma$ is a finite set. Take an exact meromorphic 1-form $\mu$ ( $\mu$ is $d f$ for some meromorphic function $f$ on $M^{\prime}$ ) on $M^{\prime}$ and a meromorphic function $g$ on $M^{\prime}$ such that restricted to $M \mu$ and $g$ are holomorphic. A sufficient condition (cf. [GK]) that $\left(\zeta^{\alpha}\right)$ given by (6) have no real periods on $M$ is

$$
g \mu \text { and } g^{2} \mu \text { have no residues and no periods on } M^{\prime} \text {. }
$$

Given that the condition (7) is met (4) defines a conformal minimal immersion

$$
\varphi: M^{\prime} \backslash \Sigma \rightarrow \mathbf{R}^{3} \text {. }
$$

The Gauss map of $\varphi$ in (8) extends holomorphically to all of $M^{\prime}$ since the $\zeta^{\alpha}$ 's involved have at worst a pole at the points of $\Sigma$. (See [L], p. 134 for a proof of this fact.)

The induced metric on $M$ is given by $h(z) d z \cdot d \bar{z}$ with $h(z)=2 \Sigma\left|\eta^{\alpha}\right|^{2}$ and the immersion $\varphi$ is complete given that

$$
\Sigma\left|\eta^{\alpha}\right|^{2}=c /|z|^{2 m}+\text { higher-order terms , }
$$

where $c \in \mathbb{C}, z$ is a local holomorphic coordinate centered at one of the points in $\Sigma$, and $\eta^{\alpha}=\partial \varphi^{\alpha} / \partial z$. The expansion shows that any path approaching one of the punctures has infinite arc length.

\section{$\S 2$. THE MAIN RESULT}

Theorem. Let $f$ be any nonconstant meromorphic function on a compact Riemann surface $M^{\prime}$ of genus $p>0$. Then there exists another meromorphic function $g$ on $M^{\prime}$ such that $\{d f, g\}$ is the Weierstrass pair giving a complete conformal minimal immersion of finite total curvature

$$
\varphi: M=M^{\prime} \backslash \Sigma \rightarrow \mathbf{R}^{3},
$$

where $\Sigma=\operatorname{supp}(f)_{\infty} \cup \operatorname{supp}(g)_{\infty}$.

Proof. Let $f$ be a nonconstant meromorphic function on $M^{\prime}$ with polar divisor

$$
(f)_{\infty}=\Sigma b_{i} p_{i} ; \quad 1 \leq i \leq n, \quad p_{i} \in M^{\prime} .
$$

Also put $d=\Sigma b_{i}$. Then $d$ is the degree of the polar divisor of $f$. And $d f$, a meromorphic 1-form on $M^{\prime}$, has poles of order $b_{i}+1$ at $p_{i}$ and no other poles. Put

$$
(d f)_{0}=\Sigma a_{j} q_{j} ; \quad 1 \leq j \leq m ; \quad q_{j} \in M^{\prime} .
$$


We then have $2 p-2=\operatorname{deg}(d f)_{0}-\operatorname{deg}(d f)_{\infty}$ since $(d f)=(d f)_{0}-(d f)_{\infty}$ is a canonical divisor. Thus

$$
\Sigma a_{j}=(2 p-2)+d+n .
$$

Define a divisor $D$ on $M^{\prime}$ by

$$
D=\Sigma a_{j} q_{j}-\Sigma c_{i} p_{i}
$$

where $\Sigma c_{i}=3 p-2+d+n$ and $c_{i} \geq b_{i}+1$. It follows that $\operatorname{deg} D=-p$. The Riemann-Roch theorem then tells us that

$$
\operatorname{dim} L(-D)=\operatorname{deg}(-D)-p+1+\operatorname{dim} L((d f)+D) \geq 1,
$$

where $L(-D)=\left\{G\right.$, meromorphic function on $\left.M^{\prime}:(G) \geq D\right\} \cup\{0\}$. Given $G \in L(-D)$ set

$$
\begin{array}{cc}
(G)_{0}=\Sigma \tilde{a}_{j} q_{j}+\Sigma \tilde{a}_{m+k} q_{m+k} ; & 1 \leq j \leq m, 1 \leq k \leq l ; \\
(G)_{\infty}=\Sigma \tilde{c}_{i} p_{i} ; & 1 \leq i \leq n .
\end{array}
$$

Note that we must have

$$
\tilde{c}_{i} \leq c_{i} ; \quad \tilde{a}_{j} \geq a_{j} ; \quad \Sigma \tilde{a}_{j}+\Sigma \tilde{a}_{m+k}=\Sigma \tilde{c}_{i} .
$$

The last condition reflects the fact that $(G)$ is a principal divisor and the first two conditions say that $G \in L(-D)$.

Define a meromorphic function $g$ on $M^{\prime}$ by

$$
g=\sum_{\alpha=1}^{\lambda} \frac{c_{\alpha}}{G^{\alpha}}
$$

where $\lambda=2(n+m+l-1)+4 p+1$. The $c_{\alpha}$ 's are complex constants to be chosen suitably later. Since $\operatorname{supp}(g)_{\infty}=\operatorname{supp}(G)_{0}$ we get

$$
\operatorname{supp}(g)_{\infty}=\left\{q_{1}, \ldots, q_{m+l}\right\} .
$$

Consider the meromorphic 1-forms $g d f$ and $g^{2} d f$ on $M^{\prime}$. Observe that

$$
\begin{gathered}
\left\{q_{m+1}, \ldots, q_{m+l}\right\} \subset \operatorname{supp}(g d f)_{\infty} \subset\left\{q_{1}, \ldots, q_{m+l} ; p_{1}, \ldots, p_{n}\right\}, \\
\left\{q_{1}, \ldots, q_{m+l}\right\} \subset \operatorname{supp}\left(g^{2} d f\right)_{\infty} \subset\left\{q_{1}, \ldots, q_{m+l} ; p_{1}, \ldots, p_{n}\right\} .
\end{gathered}
$$

We claim that we can choose $\left(c_{\alpha}\right)$, not all zero, such that $g d f$ and $g^{2} d f$ have no residues and no periods on $M^{\prime}$. Put

$$
\begin{aligned}
& R_{i \alpha}=\text { the residue of } \frac{d f}{G^{\alpha}} \text { at } p_{i}, \\
& R_{j \alpha}=\text { the residue of } \frac{d f}{G^{\alpha}} \text { at } q_{j}, \\
& R_{k \alpha}=\text { the residue of } \frac{d f}{G^{\alpha}} \text { at } q_{m+k} .
\end{aligned}
$$


So the residue of $g d f$ at $p_{i}$ is $\sum_{\alpha} c_{\alpha} R_{i \alpha}$, etc. Thus $g d f$ on $M^{\prime}$ has no residues if and only if

$$
\sum_{\alpha} c_{\alpha} R_{i \alpha}=0 ; \quad \sum_{\alpha} c_{\alpha} R_{j \alpha}=0 ; \quad \sum_{\alpha} c_{\alpha} R_{k \alpha}=0 .
$$

Now the total residue of any meromorphic 1-form must vanish. Hence

$$
\sum_{i, \alpha} c_{\alpha} R_{i \alpha}+\sum_{j, \alpha} c_{\alpha} R_{j \alpha}+\sum_{k, \alpha} c_{\alpha} R_{k \alpha}=0 .
$$

It follows that $(\mathrm{A})$ represents a homogeneous linear system in $\left(c_{\alpha}\right)$ containing at most $(n+m+l-1)$ independent equations. Let $\left(e_{1}, \ldots, e_{2 p}\right)$ be 1-cycles representing a (canonical) homology basis of $M^{\prime}$ and put

$$
P_{a \alpha}=\int_{e_{a}} \frac{d f}{G^{\alpha}} ; \quad 1 \leq a \leq 2 p, \quad 1 \leq \alpha \leq \lambda .
$$

$P_{a \alpha}$ is the $e_{a}$-period of $d f / G^{\alpha}$. So the $e_{a}$-period of the meromorphic 1-form $g d f$ is $\sum_{\alpha} c_{\alpha} P_{a \alpha}$. Thus $g d f$ has no periods if and only if

$$
\sum_{\alpha} c_{\alpha} P_{a \alpha}=0
$$

This gives a homogeneous linear system in $\left(c_{\alpha}\right)$ containing $2 p$ equations. We now consider the meromorphic 1 -form $g^{2} d f$. The residue at $p_{i}$ of $g^{2} d f$ is

$$
R_{i}\left(c_{\alpha}\right)=R_{i 2} c_{1}^{2}+R_{i 4} c_{2}^{2}+\cdots+R_{i, 2 \lambda} c_{\lambda}^{2}+2 R_{i 3} c_{1} c_{2}+\cdots+2 R_{i, 2 \lambda} c_{\lambda-1} c_{\lambda},
$$

where $R_{i, 2 \lambda}$ denotes the residue at $p_{i}$ of $d f / G^{2 \lambda}$, etc. Thus $g^{2} d f$ has no residues if and only if

$$
R_{i}\left(c_{\alpha}\right)=0 ; \quad R_{j}\left(c_{\alpha}\right)=0 ; \quad R_{k}\left(c_{\alpha}\right)=0 .
$$

Again we can eliminate one of the equations from (C) using the fact that the total residue of $g^{2} d f$ must vanish. Hence (C) represents a homogeneous quadratic system $\left(R_{i}, R_{j}, R_{k}\right.$ are all homogeneous polynomials in $\left(c_{\alpha}\right)$ of degree 2$)$ in $\left(c_{\alpha}\right)$ containing $(n+m+l-1)$ equations. Requiring $g^{2} d f$ to have no periods we obtain another homogeneous quadratic system (D) containing $2 p$ equations. The total number of equations in (A-D) is $2(n+m+l-1)+4 p=\lambda-1$ and the claim follows. (Observe that in solving the system (A-D) we are intersecting a set of hyperplanes and homogeneous hyperquadrics in $\mathbb{C}^{\lambda}$.) Equation (7) now tells us that $\{d f, g\}$ is the Weierstrass pair representing a conformal minimal immersion $\varphi: M^{\prime} \backslash \Sigma \rightarrow \mathbf{R}^{3}$, where $\Sigma=\operatorname{supp}(f)_{\infty} \cup \operatorname{supp}(g)_{\infty}=$ $\left\{p_{1}, \ldots, p_{n} ; q_{1}, \ldots, q_{m+l}\right\}$. The Gauss map of $\varphi$ extends holomorphically to all of $M^{\prime}$ since the $\zeta^{\alpha}$ 's given by (6) with $\mu=d f$ have at worst a pole at the points of $\Sigma$. Condition (9) is also routinely verified. For example, $d f$ has a pole of order $b_{i}+1$ at $p_{i}$ and condition (9) is met with $m \geq 2$.

Note that in the above proof

$$
n \leq d ; \quad m+l \leq 3 p+d+n-2 .
$$


Let $\sharp$ denote the total number of punctures of $\varphi$, i.e., $\sharp$ is the cardinality of $\Sigma$. Then we obtain

$$
\sharp=n+m+l \leq 3 p+3 d-2 .
$$

Corollary. Let $M^{\prime}$ be any compact Riemann surface of genus $p$. Then there exists a complete conformal minimal immersion of finite total curvature

$$
\varphi: M^{\prime} \backslash \Sigma \rightarrow \mathbf{R}^{3} \quad \text { with }|\Sigma| \leq 4 p+1 .
$$

Proof. Let $p_{1} \in M^{\prime}$ be a non-Weierstrass point. Then there exists a meromorphic function $f$ on $M^{\prime}$ with $(f)_{\infty}^{-}=(p+1) p_{1}$. So $n=1$. Also

$$
m+l \leq 3 p+d+n-2=4 p
$$

and the result follows.

Corollary. Let $M^{\prime}$ be any hyperelliptic Riemann surface of genus $p$. Then there exists a complete conformal minimal immersion of finite total curvature

$$
\varphi: M^{\prime} \backslash \Sigma \rightarrow \mathbf{R}^{3} \quad \text { with }|\Sigma| \leq 3 p+4 .
$$

Proof. On a hyperelliptic Riemann surface there exists a meromorphic function whose polar divisor has degree two. So we can take $d=2$.

\section{REFERENCES}

[BC] J. L. Barbosa and A. G. Colares, Minimal surfaces in $\mathbf{R}^{3}$, Lecture Notes in Math, SpringerVerlag, New York, 1986.

[CG] C. C. Chen and F. Gackstatter, Elliptische und hyperelliptische Funktionen und vollständige Minimalflächen vom Enneperschen Typ, Math. Ann. 259 (1982), 359-370.

[CO] S. S. Chern and R. Osserman, Complete minimal surfaces in Euclidean $n$-space, J. Analyse Math. 19 (1967), 15-34.

[GK] F. Gackstatter and R. Kunert, Konstruktion vollständiger Minimalflächen von endlicher Gesamtkrümmung, Arch. Rational Mech. Anal. 65 (1977), 289-297.

[HM] D. Hoffman and W. Meeks III, Complete embedded minimal surfaces of finite total curvature, Bull. Amer. Math. Soc. 12 (1985), 134-136.

[KS] T. Klotz and L. Sario, Existence of complete minimal surfaces of arbitrary connectivity and genus, Proc. Nat. Acad. Sci. 54 (1965), 42-44.

[L] H. B. Lawson, Jr., Lectures on minimal submanifolds, Publish or Perish, Berkeley, 1980. 72467

Department of Mathematics, Arkansas State University, State University, Arkansas 